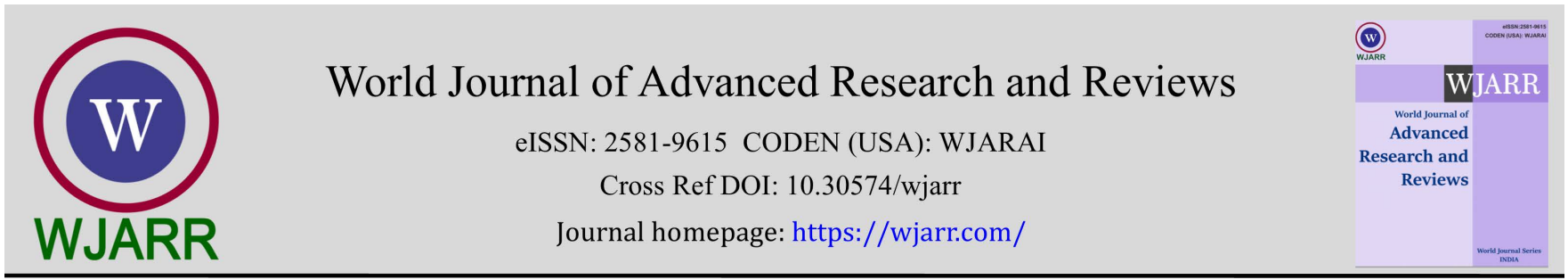

(CASE REPORT)

Check for updates

\title{
Laser treatment of the anterior capsulorhexis shrinkage syndrome in patients with pseudophakia
}

\author{
Adam Cywinski 1,* and Daniela Ferda Lewinska 1,2 \\ ${ }^{1}$ Silesian Eye Treatment Centre, Zory, Poland. \\ ${ }^{2}$ University Clinical Center prof. K. Gibinski of the Silesian Medical University, Katowice, Poland.
}

World Journal of Advanced Research and Reviews, 2021, 09(01), 250-255

Publication history: Received on 15 January 2021; revised on 22 January 2021; accepted on 24 January 2021

Article DOI: https://doi.org/10.30574/wjarr.2021.9.1.0027

\begin{abstract}
The article describes results of laser treatment applied in seven patients (eleven eyes) due to capsulorhexis margin shrinkage and the subsequent wrinkling of the anterior capsule in eyes with pseudophakia. Almost total loss of the anterior capsulorhexis aperture caused a significant decrease in visual acuity, to the level of counting fingers right in front of the eyes in three patients (four eyes). The laser cut of the anterior capsulorhexis margin using the Nd-Yag laser led to an immediate increase in the capsulorhexis size in each case. Visual acuity improved in the eyes with an extreme decrease in the capsulorhexis size. In the remaining eyes, where the decrease of the capsulorhexis size did not lead to the appearance of anterior capsule wrinkling, visual acuity did not change. The progressive decrease in the capsulorhexis size, accompanied by the appearance of white tissue on its margin, containing shrinking elements, constitutes an indication for the implementation of laser therapy.
\end{abstract}

Keywords: Anterior capsule contraction syndrome (ACCS); Posterior capsular opacification; Phimosis capsule; Pseudophakia; Cataract

\section{Introduction}

Anterior capsulorhexis, i.e. partial removal of the front part of the lens capsule, constitutes a regular part of the procedure aimed at lens removal. Through the capsulorhexis aperture, the artificial lens is inserted into the capsule, obtaining the most physiological position, both in the case of cataract removal and clear lens extraction. Properly performed capsulorhexis in terms of size and shape is one of the most important stages of the procedure, which guarantees the correct positioning of the artificial lens in the capsule. This, in turn, allows for optimal vision parameters to be obtained after the procedure. The capsulorhexis size should be adjusted to the size of optics of the artificial lens, and it ranges from 5.0 to $5.5 \mathrm{~mm}$. The size of the capsulorhexis aperture may decrease over time. In extreme cases, the capsulorhexis margin shrinks so much that the aperture in the anterior capsule is almost completely closed. Additionally, wrinkling of the anterior lens capsule appears. This unfavourable syndrome is known as the Anterior Capsule Contraction Syndrome (ACCS). ACCS was described in detail in 1993, after the introduction of continuous curvilinear capsulorhexis (CCC) to cataract surgery $[1,2]$.

The extreme shrinkage of the anterior capsulorhexis, known as phimosis, not only impairs vision. In extreme cases, the zonules of Zinn may be ruptured with subsequent subluxation, and even luxation of the capsule-artificial lens complex into the vitreous chamber [2]. Changing the capsulorhexis size adversely affects the state of postoperative vision. Excessively small capsulorhexis can cause an unwanted defect of hyperopia, caused by the shift of the optics towards the vitreous chamber. In the case of excessively large capsulorhexis, the optics shift forward, which results in the

\footnotetext{
* Corresponding author: Adam Cywinski

Silesian Eye Treatment Centre, Mailing address street: Okrezna 11, zip: 44-240, city: Zory, country: Poland. 
appearance of myopia $[3,4]$. The appearance of these unfavourable consequences is even more noticeable by the patient in the case of implantation of premium class lenses.

Reasons for shrinkage. Contrary to the migration of epithelial cells to the surface of the posterior capsule, which results in the loss of its transparency, in the capsulorhexis margin shrinkage syndrome and subsequent anterior capsule wrinkling we observe metaplasia of capsular epithelial cells and myofibroblastic transformation $[5,6,7]$. The altered cells containing actin, present in smooth muscles, are part of the white-coloured fibrous membrane visible at the capsulorhexis margin. Opacity and shrinkage of the margin of the anterior capsule appear much earlier than posterior capsule opacity (PCO), most often in the first 6 months postoperatively $[4,5]$. The lens capsule naturally shrinks when, after removing the patient's lens (thickness $\pm 4 \mathrm{~mm}$ ), it is replaced by a 2-3 times thinner artificial lens. The progression of this process occurring in all eyes that have undergone cataract removal and refractive lens replacement is limited.

There are many diseases that may predispose patients to this pathology. The most commonly described is the pseudoexfoliation syndrome (PEX). Other conditions include uveitis, retinitis pigmentosa, high myopia and myotonic dystrophy. Advanced age and eye trauma, including surgical trauma, are among other causes $(3,8)$. The abovementioned conditions, as well as post-traumatic lesions of the lens, resulting from previously performed procedures, e.g. trabeculectomy or pars plana vitrectomy, are closely related to the problem of the pathological decrease in the anterior capsulorhexis size as a result of the shrinkage of its margin. Potentially more frequent occurrence of this complication should increase the surgeon's vigilance in the case of lens surgery in the eyes with the above-mentioned eye pathologies $[9,10]$

\section{Material and methods}

Retrospective evaluation of laser treatment of anterior capsulorhexis margin shrinkage and subsequent capsular wrinkling, performed in 7 patients, including 4 binocularly. The procedures were performed in the period from March 2018 to September 2020, in eyes that had previously undergone cataract removal followed by artificial lens implantation into the capsule. In 3 patients ( 4 eyes), the shrinkage was so advanced that the post-capsulorhexis aperture was practically closed. In the remaining eyes, the capsulorhexis size was decreased in comparison with its size observed immediately after cataract removal. In each case, white fibrous tissue appeared at the margin of capsulorhexis (Figure 1)

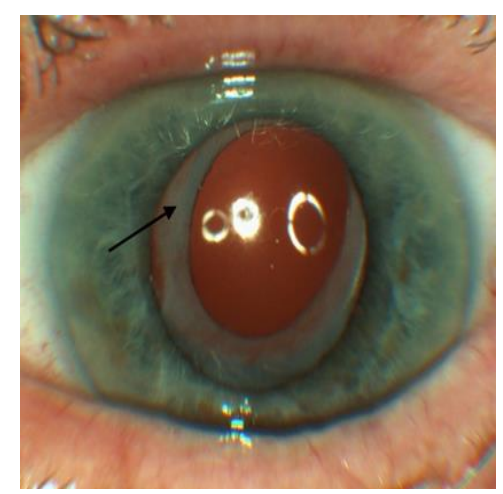

Figure $1 \mathrm{~A}$ colour photograph of the anterior segment of the eye. Pseudophakia. The visible white tissue on its edge (arrow) contains elastic fibres causing gradual capsulorhexis edge contraction with a subsequent decrease in its size.

In the eyes in which the capsulorhexis aperture was completely closed, fibrous tissue was accompanied by wrinkling of the anterior capsule (Figure 2).

The age of the patients ranged from 67 to 91 years. One of the patients (both eyes) was diagnosed with high myopia of -16.0 dioptres preoperatively.

Significant visual impairment caused that patients in whom the capsulorhexis aperture was almost completely closed came for an ophthalmological consultation. In the remaining cases, a decrease in the capsulorhexis size was observed during routine postoperative follow-up. Importantly, the changes were observed in the first weeks after cataract removal. 


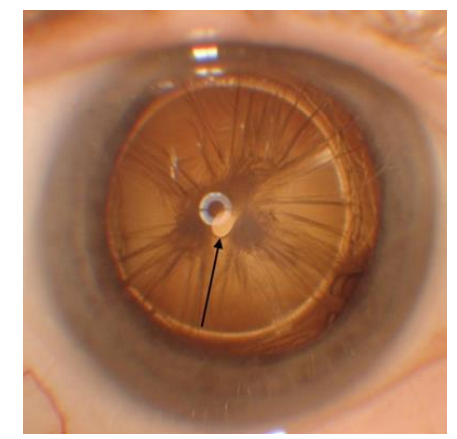

Figure 2 A colour photograph of the anterior segment of the eye. High myopia, pseudophakia. Visible significant wrinkling of the lens capsule. In the centre of the fibrous tissue there is an extremely decreased post-capsulorhexis opening (arrow).

Therefore, the patients were qualified for laser therapy with the use of the Nd-Yag laser. The procedure was performed in two versions, depending on the degree of the capsule margin shrinkage. In the case of almost complete closure of the capsulorhexis aperture, laser pulses were directed to the outer margin of the shrunk capsulorhexis margin, thus cutting the fibrous tissue from the peripheral part of the lens capsule.

In the case of a slight decrease in capsulorhexis size, radial "incisions" were made at the margin of the lens capsule, passing slightly to the periphery, beyond the fibrous tissue.

The power of the laser beam used per pulse ranged from 1.2 to $2 \mathrm{~mJ}$ and depended on the thickness of the lens capsule and the fibrous tissue.

\section{Results}

Anatomical state. Laser separation of the shrunk capsulorhexis margin from its peripheral part caused its immediate expansion (Figure $3 \mathrm{a}, \mathrm{b}$ )
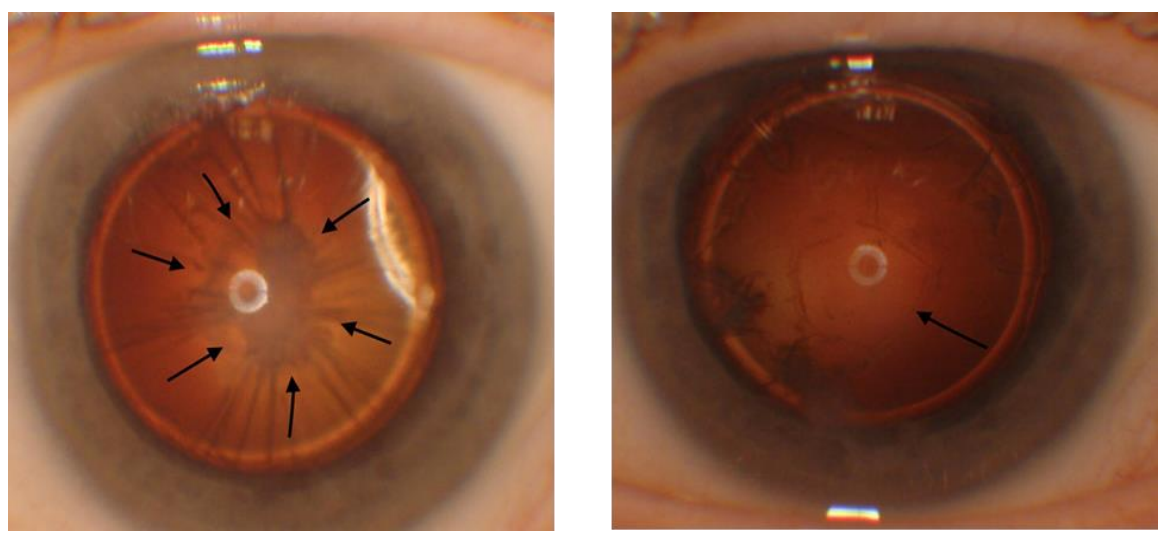

Figure 3a Colour photograph of the anterior segment of the eye. High myopia, pseudophakia. Before laser surgery. The capsulorhexis opening was practically lost. Visible wrinkles of the contracted capsule. The laser therapy line is marked with arrows. 3b. Condition after anterior and posterior laser capsulotomy (arrow). Cutting off the fibrous tissue from the peripheral part of the capsule resulted in significant expansion and widening of the capsulorhexis opening.

The same effect was obtained in the case of radial incisions at the margin of the capsule (Photograph 4a, b). The capsulorhexis aperture has considerably widened. Additionally, in the eyes with a significant capsulorhexis margin shrinkage, it was noticed that the haptics of the intraocular lens changed their non-physiological position - they no longer adhered to its optics.

Visual acuity (VA) to far distances measured in logMar. 
Before surgery, VA did not exceed the value of 2.0 in 3 patients (4 eyes) with almost complete closure of the capsulorhexis aperture. In a further 4 patients ( 7 eyes) VA ranged from

0.4 to 0.1 . The improvement of VA after the procedure occurred in the eyes in which the capsulorhexis aperture was almost completely closed, reaching values ranging from 1.5 to 0.3 . The smallest improvement in VA was observed in a patient diagnosed with high degenerative myopia who underwent the procedure in both eyes. It was 1.5 (before surgery: count fingers) and 0.7 (2.0 before surgery), respectively. The best improvement of VA from 0.8 to 0.1 was achieved in a 56-year-old man. Visual acuity did not change in the case of patients in whom the decrease in the capsulorhexis size was noticed during routine postoperative follow-up.

Visual acuity to near distances, as assessed by Snellen charts, improved in patients with extreme capsular shrinkage, in the remaining cases it remained unchanged.

\section{Discussion}

First of all, a rectification should be added. Thinking about the ACCS syndrome, the name of which appears frequently in many publications $[8,9,10]$, it should be noted that the factor initiating the appearance of wrinkling of the capsule is the anterior capsulorhexis margin shrinkage. Thus, we should be talking about the anterior capsulorhexis margin shrinkage syndrome and its consequences.

When analysing the obtained results of laser treatment, two basic conclusions can be drawn. The first is the high efficiency of laser therapy after using the Nd-Yag laser in the eyes with extreme capsulorhexis margin shrinkage and closure of the capsulorhexis aperture. Vision was improved in each case. Similar results were obtained by Hoon Dong Kim et al. and Sunil P Deokule et al. [11, 12].

Performing the procedure in the eyes in which a decrease of the capsulorhexis size was noticed during routine postoperative follow-ups did not result in the improvement of vision, but only (or yet so much) an increase in the size of the capsulorhexis aperture. Therefore, it would be necessary to consider when laser treatment should be performed. Should the eye be observed and laser therapy initiated only when vision deteriorates due to the anterior capsulorhexis margin shrinkage? Deterioration of vision occurred in eyes with anatomical changes in the lens-capsule complex, i.e. decreased capsulorhexis size and wrinkling of the anterior capsule, which led to a significant stress on the zonules of Zinn. This consequently led to a change in the position of the artificial lens in the capsule. The appearance of such a condition is not beneficial for the patient.

Assuming the principle of the superiority of prevention over treatment, it is best to start laser therapy when you notice a significant decrease in the size of capsulorhexis aperture. To diagnose it, it is enough to compare the size of the optics of the artificial lens and the size of the capsulorhexis aperture. In the case of its decrease, laser incisions of the capsulorhexis margin constitute sufficient therapy, after which an immediate increase in the capsulorhexis size is observed (Figure $4 \mathrm{a}, \mathrm{b})$.
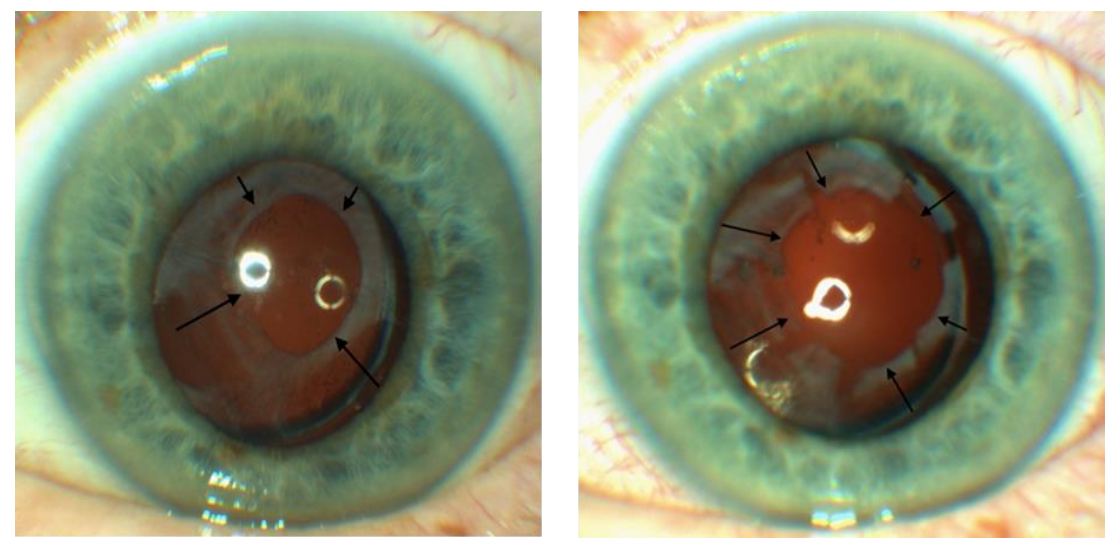

Figure 4a Colour photograph of the anterior segment of the eye. High myopia, pseudophakia. Significantly decreased size of capsulorexia due to the contraction of its edge (arrow). Distance visual acuity 2.0 logMar. 4b. Condition after radial laser incision of the caspulorhexis edge. The size of capsulorhexis was significantly increased (arrows) due to the expansion of the lens capsule. Distance visual acuity $=0.7 \log$ Mar. 
Excessively long delay in the implementation of therapy may consolidate the abnormal anatomical state and thus prevent the expansion of the lens capsule after the procedure, with all its consequences (Figure 5).

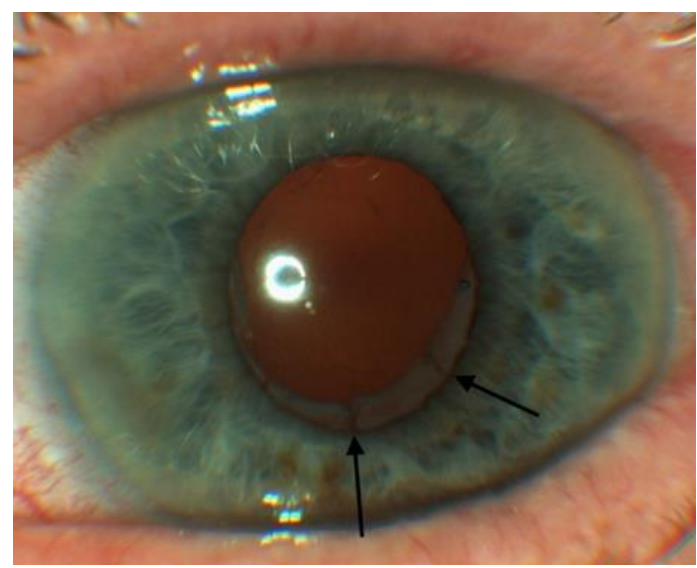

Figure 5 A colour photograph of the anterior segment of the eye. Sites after laser incision of the edge of anterior capsulorhexis (arrows), 15 months after cataract removal. The size of capsulorhexis did not change significantly.

In order to obtain a good, long-lasting visual improvement after lens removal in eyes with an increased risk of the anterior capsulorhexis margin shrinkage syndrome (including PEX syndrome), it is recommended that the size of capsulorhexis aperture should be only slightly smaller than the size of the optics of the artificial lens [12,13]. Additionally, removal of the epithelium from the anterior capsule should be considered. Some authors note that this procedure reduces the risk of capsular opacification and capsulorhexis margin shrinkage [14, 15].

The shape of the optics does not affect the prevalence of capsulorhexis margin shrinkage and of capsule contraction [16, 17]. One might suppose that the introduction of a capsular tension ring (CTR) would reduce the risk of shrinkage, but subsequent experiments have shown that this is not the case. The introduction of a CTR does not guarantee such protection, especially in cases of the pseudoexfoliation syndrome. Some authors described the appearance of subluxation or luxation of the artificial lens-capsule complex many years after cataract removal [18].

\section{Conclusion}

The capsulorhexis margin shrinkage, resulting in a decrease in the capsulorhexis size, and even more so, complete closing of the capsulorhexis aperture should be treated with the Nd-Yag laser. The procedure is not always associated with the improvement of vision, but in every case it allows us to stop the process of a decrease in the capsulorhexis size. The implementation of laser therapy should not be postponed until the patient's vision deteriorates. The decrease in visual acuity indicates the appearance of significant anatomical disorders in the position of the lens in the capsule,that are sometimes irreversible. The appearance of white tissue on the capsulorhexis margin is most often associated with the initiation of the shrinkage process. This condition requires special attention and more frequent ophthalmic appointments. Diagnosing dynamic progression of the above lesions constitutes an indication for the implementation of laser therapy.

\section{Compliance with ethical standards}

\section{Disclosure of conflict of interest}

Authors declare no competing interest.

\section{Statement of informed consent}

Informed consent was obtained from all individual participants included in the study.

\section{References}

[1] Wilde C, Ross A, Awad M, Chen HC, Dua HS. Management of anterior capsular contraction syndrome: pitfall of circular capsulotomy technique with the neodymium YAG laser. Eye (Lond). 2018; 32(9): 1546-1548. 
[2] Davison JA. Capsule contraction syndrome. J Cataract Refract Surg. 1993; 19: 582-589.

[3] Masket S. Postoperative complications of capsulorhexis. J Cataract Refract Surg. 1993; 19: 721-724.

[4] Hanson S, Crandall A, Olson R. Progressive constriction of the anterior capsular aperture following intact capsulorhexis. J Cataract Refract Surg. 1993; 19: 77-82.

[5] Gimbel HV, Neuhann T. Continuous curvilinear capsulorhexis (letter). J Cataract Refract Surg. 1991; 17: 110-111.

[6] Caporossi A, Casprini G, Toss GM, et al. Histology of anterior capsule fibrosis following phacoemulsification. J Cataract Refract Surg. 1998; 24: 1343-1346.

[7] Kurosaka E, Ando I, Kato K, et al. Fibrous membrane formation at the capsule margin in capsule contraction syndrome. J Cataract Refract Surg. 1999; 25: 930-935.

[8] Werner L, Pandey S, Escobar-Gomez M, et al. Anterior capsule opacification: a histopathological study comparing different IOL styles. Ophthalmology. 2000; 107: 463-471.

[9] Gallagher SP, Pavilack MA. Risk factors for anterior capsule contraction syndrome. J Cataract Refract Surg. 1999; 25: 1356-1361.

[10] Werner L, Pandey S, Apple DJ, et al. Anterior capsule opacification: correlation of pathologic findings with clinical sequelae. Ophthalmology. 2001; 108: 1675-1681.

[11] Long-term follow-up of neodymium: YAG laser anterior capsulotomy for the treatment of anterior capsular phimosis. Hehua Ye, Jiming Zhang, Yiyong Qian .J Int Med Res. Sep 2018; 46(9): 3692-3697.

[12] Complete occlusion of anterior capsulorhexis after uneventful cataract surgery, treated with YAG laser capsulotomy. Hoon Dong Kim, Jae Min Kim, Jong Jin Jung. BMC Ophthalmol. 4 Dec 2017; 17(1): 233.

[13] Neodymium:YAG laser anterior capsulotomy for capsular contraction syndrome. Sunil P Deokule, Subhanjan $\mathrm{S}$ Mukherjee, Chris K S Chew. Ophthalmic Surg Lasers Imaging. Mar-Apr 2006; 37(2): 99-105.

[14] Abela-Formanek C, Amon M. Uveal and capsular biocompatibility of hydrophilic acrylic, hydrophobic acrylic, and silicone intraocular lenses. J Cataract Refract Surg. 2002; 28: 50-61.

[15] Kato S, Suzuki T, Hayashi Y, et al. Risk factors for contraction of the anterior capsule aperture. J Cataract Refract Surg. 2002; 28: 109-112.

[16] Ahmed I, Chen, S, Kranemann C, Wong DT. Surgical repositioning of dislocated capsular tension rings. Ophthalmology. 2005; 112: 1725-1733.

[17] Lim M, Jap A, Wong E. Surgical management of late dislocated lens capsular bag with intraocular lens and endocapsular tension ring. J Cataract Refract Surg. 2006; 32: 533-536.

[18] Deka S, Deka A, Bhattacharjee H. Management of posteriorly dislocated endocapsular tension ring and intraocular lens complex. J Cataract Refract Surg. 2006; 32: 887-889. 